
allemande

50-2 | 2018

Humanités environnementales - Quoi de neuf du côté des méthodes?

\title{
La sociologie à l'épreuve de l'altérité
}

Esquisse d'une grammaire de terrain

Christophe Baticle, Florence Rudolf, Anahita Grisoni et Sophie Némoz

\section{(2) OpenEdition}

\section{Journals}

Édition électronique

URL : https://journals.openedition.org/allemagne/844

DOI : 10.4000/allemagne.844

ISSN : 2605-7913

Éditeur

Société d'études allemandes

Édition imprimée

Date de publication : 30 décembre 2018

Pagination : 239-253

ISSN : 0035-0974

Référence électronique

Christophe Baticle, Florence Rudolf, Anahita Grisoni et Sophie Némoz, « La sociologie à l'épreuve de l'altérité », Revue d'Allemagne et des pays de langue allemande [En ligne], 50-2 | 2018, mis en ligne le 30 décembre 2019, consulté le 19 mai 2021. URL : http://journals.openedition.org/allemagne/844; DOI https://doi.org/10.4000/allemagne.844 


\section{La sociologie à l'épreuve de l'altérité \\ Esquisse d'une grammaire de terrain}

\section{- Christophe Baticle, Florence Rudolf, Anahita Grisoni, Sophie Némoz*}

Contrairement aux sciences de la nature, qui ne partagent pas une commune humanité avec les réalités qu'elles interrogent, les sciences humaines et sociales travaillent sur des «objets» qui impliquent des «sujets", amenant ces disciplines à se faire accepter pour pouvoir accéder à leurs terrains. Ainsi, toute investigation sociologique partage une épreuve commune, qui a trait à sa négociation au sein d'une configuration dont elle ne fait pas initialement partie, et qui influe sur les questions investiguées, l'approche méthodologique retenue et in fine les propositions de connaissances produites. Si cette prise de conscience de l'obligation pour les sciences sociales de «faire avec» n’a pas suivi les mêmes cheminements selon les courants de pensée, l'accommodement au terrain s'avère de nos jours un passage obligé de la pratique sociologique. Cette condition prévaut, y compris lorsqu'il a été fait explicitement appel à une expertise sociologique ou que la démarche opère à partir d'une situation d'immersion (que ce soit à couvert ou non) voulue par le chercheur. Ces deux cas limites (commande et immersion incognito) définissent un intervalle de possibles à partir desquels le savoir sociologique se construit au gré des négociations entre des acteurs différemment impliqués à la construction de représentations sociales susceptibles d'influer le cours des choses. Dans tous ces cas, l'implication des sociologues sur un terrain participe de la production d'un double (une nouvelle représentation de la réalité, une problématique qui renouvelle le questionnement) qui interpelle les situations sociales. Ce double, issu des résultats de l'analyse sociologique, fonctionne comme un miroir, fidèle ou déformant, à partir duquel les protagonistes des situations sociales peuvent

* Respectivement: post-doctorant, socio-anthropologue, Université de Picardie Jules Verne, EA 4287 «Habiter le monde»; professeure des universités en urbanisme, Institut national des sciences appliquées de Strasbourg, directrice adjointe de l'EA 7309 AMUP; chercheure affiliée à I'UMR 5600 «Environnement, Ville, Société», Université de Lyon; et maître de conférences en sociologie-anthropologie, Université de Bourgogne/Franche-Comté, UFR des sciences du langage, de l'Homme et de la société. 
ou non se reconnaître. La présence du chercheur fait écho à la figure de l'étranger que Simmel associe à l'irruption d'une prise de distance possible au sein des configurations sociales, autrement dit à l'émergence d'un potentiel critique. Cette posture contribuerait à la généralisation de l'objectivation et de la montée en généralité, propre à la modernité contemporaine. Le questionnement, ici soulevé, concerne ainsi les effets provoqués par la démarche sociologique sur ces configurations sociales, mais également et en retour les implications de ce processus sur le travail sociologique.

Le double remplit une fonction d'observation à laquelle œuvrent différents acteurs, au premier chef desquels les protagonistes investis dans la production d'un rapport de distanciation au monde. En conséquence, on a toujours affaire à au moins un acteur de terrain, voire à une coalition d'acteurs, qui tente d'enrichir une situation sociale d'une nouvelle dimension, apportée par la compétence sociologique. Cette situation, que l'on peut également qualifier de «réflexive», constitue une épreuve sociale dont on mesure l'importance à l'ensemble des transformations qui caractérisent la modernité. Un des enjeux et non des moindres d'une démarche d'investigation sociologique débute par l'accueil que le terrain réserve au chercheur (ou à l'équipe de chercheurs). Selon que ce dernier est prêt à composer et selon les modalités de cette composition, la recherche peut prendre différentes directions. Avec la démocratisation des sciences et des techniques, l'engagement du terrain dans la production des connaissances se généralise et avec lui les exigences posées aux chercheurs. Cette situation ouvre la voie à toute une gamme de relations qui justifie qu'on y prête attention. L'enjeu sera redoublé avec l'essor des humanités environnementales qui met au défi d'entrer en recherche avec: primo des entités qui ne partagent pas une commune humanité (animaux, phénomènes hybrides entre nature et culture) et secundo avec des protagonistes (scientifiques, praticiens et ingénieurs naturalistes) qui ne sont pas familiers des sciences sociales.

De la sorte, l'objet de cet article vise à répertorier les cadres sociaux impliqués dans la production de connaissances sociologiques afin de rendre compte de la diversité et de la complexité de l'entreprise sociologique à mesure que la société s'ouvre à la réflexivité et que les non-humains accèdent à une reconnaissance, sinon de sujets, de partenaires de la connaissance et de la composition de mondes communs ${ }^{(1)}$. Selon une première typification qui nous sert de guide, nous distinguons les situations dans lesquelles les chercheurs se fondent dans leur terrain et s'y confondent (situation d'immersion) et celles pour lesquelles ils passent pour des «étrangers» au sens de Simmel ${ }^{(2)}$ (différentes postures que nous développons ci-dessous sont possibles). Cela nous amène à observer les relations des chercheurs à leurs terrains sous l'angle de figures typiques et de grammaires sociales en évolution par rapport auxquelles nous formulons l'hypothèse qu'elles conditionnent la démarche d'enquête, la collecte d'informations et la construction de données, mais également la dynamique sociale elle-même. Selon les modalités d'entrée dans le terrain et les capacités à négocier des transitions, nous postulons des

1 Laurence Boutinot, Christophe Baticle, "Surveiller sans punir, "discrètement". Un commun de résistance au travers du "braconnage” dans les forêts camerounaises", Espaces et sociétés, 2018 (à paraître).

2 Georg Simmel, «Digression sur l'étranger» (1908), in: Yves Grafmeyer, Isaac Joseph, L'École de Chicago. La naissance de l'écologie urbaine, Paris, Aubier (coll. Champ Urbain), 1979, p. 53-77. 
différences en matière de construction du savoir sociologique et en matière de projet. Nous aspirons de la sorte à poser les linéaments d'une grammaire sociale complexe à partir de la figure inaugurale et idéal-typique de l'«étranger» en sociologie ${ }^{(3)}$. Cette dernière définit deux grands cas, selon qu'on est ou non un protagoniste d'une situation sociale (in ou outsider).

\section{De l'idée du double à la double herméneutique : la pratique sociologique en action}

Dans une approche alliant empathie et démarche critique, cette dimension réflexive peut se donner pour tâche de contribuer à la compréhension du terrain par les acteurs, mais aussi à la mise en lumière des situations de domination qui y sont inhérentes. C'est notamment le cas dans la sociologie des mouvements sociaux, qui, à travers ses objets, se donne pour tâche la construction d'un autre regard sur les luttes sociales ${ }^{(4)}$. Cette double épreuve, que le sociologue contemporain Anthony Giddens qualifie de double herméneutique ${ }^{(5)}$, participe d'une transformation sociale par la connaissance. La transformation opère par un travail de mise en signification quant au sens du contexte qui se déploie socialement, c'est-à-dire à travers différentes couches et situations sociales. Ce couplage du chercheur au terrain et réciproquement rend compte des enjeux de négociation inhérents à des situations de recherche: négocier sa place de tiers dont les énoncés et propositions de sens seront ou non repris et mis en action par différents acteurs dans des configurations diverses. Cette dynamique est productrice de métadonnées quant à la structure du collectif qui se prête à ce jeu de réflexivité. En référence à Robert D. Putnam ${ }^{(6)}$, on pourrait qualifier les terrains selon qu'ils se caractérisent par leur ouverture bridging capital ou leur fermeture bonding capital. Ces capitaux étant aussi des ressources que les chercheurs parviennent ou non à mobiliser pour négocier leur place au sein de collectifs qui leur rendent la tâche plus ou moins aisée.

\section{L'«étranger» comme figure idéal-typique de la situation de recherche}

Nous avançons à partir de l'hypothèse d'une imbrication serrée entre l'investigation sociologique qui fait écho à la figure de l'étranger et le travail d'hospitalité d'un collectif à l'égard d'un tiers. Dans son essai sur l'étranger, Simmel explore un type de relation sociale où la distance l'emporte sur la proximité contrairement à celle qui caractérise les familiers. L'apport de Simmel réside ainsi dans les atouts, dont il rend compte, de la prédominance de la distance sur la proximité. La figure de l'étranger qui se généralise avec certaines situations sociales, comme dans les villes notamment, permet d'explorer les avantages de la distance qui rime avec montées en généralité, compétences en matière d'abstraction et de typification. Non intégré au jeu social qui prévaut sur le terrain qu'il explore, l'étranger est ainsi davantage susceptible de

3 L'étranger selon Simmel renvoie à l'irruption du tiers producteur de réflexivité sociale.

4 Anahita Grisoni, Sophie Némoz, "Les mouvements sociaux écologistes: entre réforme de soi et rapports de classe, entre histoires nationales et circulations européennes », Socio-logos (en ligne), 12 (2017), http://socio-logos.revues.org/314.

5 Anthony Giddens, Les conséquences de la modernité, Paris, L’Harmattan, 1994.

6 Robert D. Putnam, Bowling Alone: The Collapse and Revival of American Community, New York, Simon and Schuster, 2001. 
recueillir les confidences des acteurs sans que ces derniers ne se sentent menacés par ce partage. Alors que le secret, le non-dit et le quant à soi s'imposent avec des proches, en raison de ce qu'ils sont directement concernés par les états d'âme, les intentions et motivations de leurs congénères, l'étranger offre des prises pour des confidences. L'échange permet de rendre compte des situations, d'en saisir les logiques. Il participe d'un processus d'objectivation. Ainsi, sur des terrains dont la pérennité même du collectif dépend dans une certaine mesure du maintien du secret quant aux activités d'une partie de ses membres, le sociologue extérieur et discret accueillera plus facilement les témoignages qu'un membre de la société locale.

C'est le cas notamment des groupes dont certains membres agissent en marge de la loi. L'exemple d'une enquête auprès de thérapeutes exerçant dans le domaine des médecines non conventionnelles atteste de l'attractivité que peut constituer la présence d'un tiers. Ce dernier, un acteur d'un type un peu particulier parce qu'impliqué à une autre échelle que les autres, offre un plan d'observation et d'action supplémentaire à la configuration d'acteurs qu'il a rejointe. L'absence de réglementation et de cadre juridique de la part de l'État implique l'existence de frontières légales agissant "par défaut» sur les praticiens, pouvant être accusés d'exercice illégal de la médecine, de la pharmacie ou de la kinésithérapie ${ }^{(7)}$. En vue d'une reconnaissance légale de certaines de ces techniques du corps ou médecines non conventionnelles - c'est notamment le cas de la naturopathie -, une partie des praticiens et des directeurs de centres de formation portant ce projet a encouragé, accepté ou parfois toléré la présence de la sociologue étrangère à ce milieu dans tous les espaces sociaux de son expression: domicile des soignants ou centres de soins, espaces de vente de produits bio, congrès professionnels, etc. Dans le contexte d'une compétition individuelle sur le marché du soin naturel - puisque la naturopathie se limite à une prestation de service - la figure du sociologue étranger aux règles et aux enjeux de cette profession en devenir devait permettre l'introduction de cette médecine non conventionnelle, quoique par une entrée critique, à l'université.

Si le texte de Simmel ouvre la réflexion au travail d'hospitalité réservé à l'étranger en conclusion de son essai, il faudra attendre le texte d'Isaac Joseph «Prises, Réserves, Épreuves » ${ }^{(8)}$ pour une exploration toute en finesse des dispositions sociales et aménagements spatiaux nécessaires à l'épanouissement d'une telle figure. À la manière dont proximité et distance sont en jeu dans «Digression sur l'étranger», prises et déprises se répondent dans ce texte pour réserver un juste accueil à l'étranger. Selon l'esprit de cet article, un terrain accueillant offrirait des prises spatiales tout en ménageant $\mathrm{du}$ jeu aux mouvements et aux initiatives, d'une part, et témoignerait d'une reconnaissance sociale sans en faire trop, d'autre part. Les va-et-vient empiriques invitent à questionner la «recherche de plein air» telle que la sociologie des sciences définit ce mouvement de convergence des savoirs ${ }^{(9)}$.

7 Anahita Grisoni, Sous les pavés, la terre. Entre culte du bien-être et loi du marché, la naturopathie en expansion sur l'espace public, thèse de sociologie soutenue le 31 janvier 2011, EHESS, Paris.

8 Isaac Joseph, «Prises, Réserves, Épreuves», Communications, nº 65 (1997), p. 131-142.

9 Michel Callon, Pierre Lascoumes, Yannick Barthe, Agir dans un monde incertain. Essai sur la démocratie technique, Paris, Seuil, 2001. 


\section{Hybridation de la figure de l'«étranger» et démultiplication des grammaires sociales}

Cet élargissement des collectifs de recherche, dont résulte de fait une imbrication croissante des communautés de pratiques, témoigne de ce que la figure de l'étranger n'est plus la norme absolue en sociologie en raison de sa propension à l'hybridation. Elle a servi de guide et de référent pour penser les effets de l'urbanisation spectaculaire des métropoles consécutivement aux grandes migrations du $\mathrm{XIX}^{\mathrm{e}}$ et du XX $\mathrm{XX}^{\mathrm{e}}$ siècle. Pour paraphraser l'École de Chicago, la socialisation des migrants rime avec l'expérience de la déstructuration sociale au quotidien. Il s'ensuit des relations à géométrie variable qui gagneraient à être davantage documentées pour un approfondissement des correspondances entre conditions d'enquête et production des connaissances, voire en vue d'une évaluation des propositions de recherche, hypothèses et thèses comprises. Qu'en est-il aujourd'hui en sociologie de l'environnement?

Depuis plus de dix ans de recherches-actions et de recherches-formations ${ }^{(10)}$ sur des problématiques dites "environnementales», nous interrogeons les ressorts de collectifs de recherche qui croisent des publics distincts ${ }^{(11)}$. Qu'il s'agisse de pratiques pédagogiques ou de conduite de projets, ces expériences nous renseignent sur la richesse des situations sociales et nous inspirent l'écriture d'une grammaire de la recherche en action.

Que ce soit sur des terrains pacifiés ou au contraire minés par des conflits de longue date, l'exhumation du passé-présent, de la préparation d'une manifestation comme les Journées de l'architecture ${ }^{(12)}$ jusqu'à l'étude d'un projet urbain, réserve bien des surprises. C'est ainsi que le travail sur le campus de l'Esplanade à Strasbourg, en amont de la rénovation urbaine accompagnant l'opération Campus, en 2009, a fait resurgir la mémoire des luttes et occupations étudiantes ${ }^{(13)}$. Sur un autre site, celui du centre commercial, à deux pas du campus étudiant, c'est au contraire une forme de sociabilité plus éclatée qui s'est imposée par les itérations entre équipes d'artistes, de chercheurs et de citadins.

Sur la portée d'une telle expérience, l'analyse des interlocutions entre étudiants, enseignants-chercheurs, mais aussi des participants qui ne sont pas issus du milieu universitaire, nous apprend peut-être bien davantage quant à l'implicite et de l'explicite

10 Sophie Némoz, «Vers une énergie intelligente pour la mobilité universitaire? Le cas de la rechercheaction "Smart Campus" ", VertigO - la revue électronique en sciences de l'environnement, 14/3 (2014): dossier "Transition énergétique: contexte, enjeux et possibilités», http://vertigo.revues.org/15845; Sophie NÉMOZ, «La recherche de "plein air" à la campagne: conférence-débat sur les pratiques sociales du climat et les perspectives d'éco-innovation territoriale», Conference of Youth (COY 11), 11e édition de la Conférence de la jeunesse, Villepinte, 27 novembre 2015.

11 Étudiants, enseignants-chercheurs et praticiens.

12 Véronique Ejnès, Florence Rudolf, «Espla, années 70. Jeux de résonnance», Les journées de l’architecture, Strasbourg, 2010 ; Véronique Ejnès, Florence Rudolf, «Du campus au quartier, la ville est là », Les Journées de l'architecture, Strasbourg, 2012.

13 Florence Rudolf, "Campus - Démocratiser les œuvres architecturales et urbaines», in: Cristiana Mazzoni, Andreea Grigorovschi (éd.), Strasbourg métropole. Ourlets urbains dans la ville-mosaïque, Paris, La Commune, 2014, p. 135-148; Florence Rudolf, «Démocratiser les œuvres architecturales et urbaines », Questions de communication, n 25 (2015), Anne MASSERAn (éd.), p. 65-78. 
de la pratique ethnographique de l'environnement ${ }^{(14)}$. L'étranger pour Georg Simmel est une figure mobile, susceptible de s'installer sur un territoire sans intégrer les communautés. Certains sociologues, comme à l'instar de Pierre Bourdieu dans La misère $d u$ monde ${ }^{(15)}$, lui ont préféré un «indigène», formé par ses soins, au nom justement de la nécessité de rompre avec toute forme d'étrangeté. Les temporalités des enquêtes de terrain et des projets environnementaux sont différemment appréhendées. Dits et non-dits, les cadres temporels impliqués dans la production de connaissances et d'actions environnementales requièrent un travail continu de négociation. Entre hostilité et hospitalité, la confrontation à des formes imprévues et imprévisibles éprouve le principe de réciprocité.

Sur un autre terrain, celui du littoral picard miné par des conflits territoriaux entre les occupants séculaires que sont les chasseurs et leurs outsiders environnementalistes, la position de tiers scientifique est littéralement sabotée par les assignations indigènes qui enjoignent le chercheur à se situer comme partisan ou détracteur ${ }^{(16)}$. Mais, à l'opposé de la posture du chercheur en surplomb, loin de l'apesanteur d'une tour d'ivoire, l'enquête de terrain amène le socio-anthropologue à intervenir sur un terrain qui lui fait prendre conscience de sa culture de la nature, plus ou moins en phase avec celle des parties prenantes. Selon une posture similaire, Jean Jamin ${ }^{(17)}$, dans son travail de jeunesse sur les tenderies aux grives de ses Ardennes natales, se trouva ainsi amené à interroger son rôle au sein de cette configuration conflictuelle, mais encore ses propres incompréhensions.

Comme on peut le constater au travers de ces deux derniers exemples, les cas pour lesquels la posture du tiers est chahutée ne manquent pas et témoignent de ce que la figure de l'étranger est davantage un horizon, une figure de style qui sert à penser en-dehors des cadres hérités propres à la communauté et à l'entre soi. Elle est une figure qui permet de se projeter dans d'autres constitutions sociales, à l'image de la distinction célèbre qui substitue l'association à la communauté(18). Elle demeure pertinente pour rendre compte de la situation de recherche en ce que cette dernière correspond toujours à une inversion du rapport entre proximité et distance qui prévaut au sein d'un groupe et d'une situation sociale. Ce qui domine au quotidien, soit la reproduction des pratiques et du groupe, devient second pour la recherche qui vise

14 Sophie Némoz, «L’interdisciplinarité des savoirs face aux conséquences inattendues de l'éco-innovation", A. Coulbaut-Lazzarini, S. Némoz (dir.), L'éco-innovation au prisme du développement durable. Regards et contributions des sciences sociales, Paris, L'Harmattan (Questions contemporaines), 2013, p. 73-84.

Pierre Bourdieu (dir.), La misère du monde, Paris, Seuil, 1993.

Christophe BATICLE, Les pratiques de chasse comme affirmations politiques du principe d'autochtonie. Dimensions territoriales des luttes cynégétiques. Études de cas de la Picardie, Savoie, Normandie et Pyrénées, thèse de doctorat en socio-anthropologie, sous la direction de Jean Copans et de Bernard Kalaora, Amiens, Université de Picardie Jules Verne, 2007, 950 p.; Christophe BAtiClE, «Le tiers scientifique, nouvel acteur dans les conflits de nature entre chasseurs et environnementalistes ", Négociations: conflit, décision et délibération, $\mathrm{n}^{\circ} 24$ (2015), p. 117-130.

17 Jean Jamin, «Deux saisons en grivière. De la tradition au délit de tradition », Études rurales, numéro spécial 87-88: «La chasse et la cueillette aujourd'hui», juillet-décembre 1982, p. 41-62. 
à l'intelligibilité des structures qui se reproduisent et se transforment au gré de ces pratiques ${ }^{(19)}$. L'irruption d'un chercheur dans une configuration sociale correspond en effet à une perturbation d'un type assez singulier dans la mesure où son intéressement et concernement pour un collectif et une cause sont seconds ou médiés par l'intérêt de connaissance. Pour autant, le chercheur n'échappe pas à la logique du don et du contre-don: pour recevoir il doit également fournir des données quant à ses options personnelles, lorsque ce ne sont pas ses hypothèses voire ses données de terrain qui sont soumises à questionnement et à contrôle ${ }^{(20)}$. Les épreuves et transitions négociées par les sociologues en situation de recherche sont autant d'informations sur des postures sociologiques que sur des logiques sociales en vigueur dans différents collectifs.

Ce contre-don peut faire l'objet d'une négociation aux racines éthiques. Enquêtant sur le mouvement d'opposition à la ligne ferroviaire entre Lyon et Turin, Anahita Grisoni s'est retrouvée confrontée à une situation délicate d'usage des données de première main. Son statut de chercheure contractuelle dans un laboratoire essentiellement composé de géographes (l'UMR Environnement, Ville, Société, de l'université de Lyon) lui avait permis d'assister à une conférence organisée par la Caisse des dépôts et consignations en mars 2014, et portant sur le financement des projets de grandes infrastructures de transports dans le cadre du réseau transeuropéen de transport. Contacté à l'issue de l'événement, son organisateur à la Caisse des dépôts et consignations avait nié le caractère public de la réunion et avait demandé à la sociologue de ne pas diffuser les enregistrements. Or, un militant actif du côté français du mouvement avait insisté dans le sens contraire, afin que les fichiers audio soient prêtés à un journaliste identifié par lui et publiés dans la presse.

Sur son terrain caussenard (Lozère et Aveyron), Christophe Baticle ${ }^{(21)}$ est confronté pour sa part à une véritable ethnographie de la sauvegarde. Ses interlocuteurs, des édiles cynégétiques, acceptent le contrat moral d'une investigation autonome et objectivante, tout en ne cachant rien de leurs espoirs de "sauver les tendelles». Cette pratique de piégeage des turdidés, au moyen d'un système de pierres fonctionnant comme un assommoir, est en effet menacée de disparition puisque soumise depuis 2005 au principe du «bouilleur de cru», une liste nominative de pratiquants étant autorisée à poursuivre leur vie durant, sans possibilité de renouvellement. Pour les partisans de la «tradition" caussenarde, la patrimonialisation de la démarche reste la seule porte de salut, au travers notamment du classement de l'agropastoralisme méditerranéen en tant que Bien de l'UNESCO. Mais les résultats de l'enquête, sans être contraires à cette visée, apporteront des conclusions étonnantes qui mettront en avant le jeu des acteurs en présence. En effet, les dits «prélèvements» sur la population des turdidés sont d'une part dérisoires et ne menacent en rien la pérennité de ces espèces. D’autre part, le

19 L'étranger cultive la distance là où les membres d'un collectif cultivent la proximité et inversement. Se pose néanmoins la question de la zone de flottement entre cette démarche et les différentes formes d'intervention sociale, de recherche-action ou de recherche-action collaborative.

20 Maïté BoullosA-Joly, «Doit-on militer aux côtés des Indiens? Les risques de l'instrumentalisation du chercheur en milieu militant», Sciences humaines et sociales, n 4 (2013): «Brésil(s)», p. 125-147.

21 Christophe Baticle, La grive et le genévrier: habiter en caussenard(e). "Gardarem los tindelles " sur les Grands Causses de Lozère et d'Aveyron, rapport d'étude, Amiens, UPJV, 2016, 200 p. 
dispositif qui, à partir d'un système de cales et de tranchées échappatoires, a rendu cette pratique «sélective», permettant de la rendre acceptable au regard de la directive européenne Oiseaux (79-409), n'était qu'un moyen de contourner les représentations peu valorisantes que le Ministère de l'environnement nourrissait à l'endroit des tendeurs: de vieux messieurs issus d'espaces ruraux relégués et d'une paysannerie d'un «autre âge». On a ainsi placé un paravent de matérialité devant la véritable méthode de sélectivité, toute contenue dans le doigté de ces experts en tension qui, au travers de leurs doigts grossis par une vie de travail manuel, ont acquis cette compétence: «tendre» avec suffisamment de «finesse» pour ne faire tomber la pierre mortelle que sous la pression d'un oiseau et ce en fonction de son poids, jusqu'à quelques dizaines de grammes près. Ainsi, ces paysans «mal dégrossis», que plusieurs associations de protection de la nature avaient décrits sous des termes peu flatteurs, se révélaient fort habiles, s'ils le souhaitaient, pour éviter la prise de passereaux protégés. D'étranger suspect de faire le jeu des «écolos», le sociologue devenait alors un acteur involontaire de la sauvegarde d'une «tradition».

La figure de l'étranger n'est pas la seule à faire l'expérience de sa remise en question par le terrain. Une autre figure emblématique de l'expérience sociologique, celle du chasseur de mythe ${ }^{(22)}$, est également concernée. Les sociologues croisent de plus en plus souvent sur leurs terrains des acteurs réflexifs qui prétendent aux mêmes compétences qu'eux et défendent leur position d'autorité. Il peut s'agir d'une personne formée à la sociologie, impliquée par sa principale raison sociale sur le terrain où s'effectue la recherche, mais aussi d'autres compétences sociales en raison des multiples formes d'expertise qui accompagnent les pratiques professionnelles. Ce cas limite se rencontre plus souvent qu'on ne le pense en raison des nombreux acteurs réflexifs susceptibles d'agir dans un domaine d'action donné. La généralisation de l'intervention sociale par le projet complique le travail de négociation des chercheurs. L'activité de recherche sociologique se trouve de plus en plus en compétition avec les porteurs de projets en raison de ce que leurs compétences s'apparentent de plus en plus à celles des chercheurs. La généralisation de la double herméneutique comme forme privilégiée de l'intervention sociale met les chercheurs en porte-à-faux avec les acteurs. La confrontation de différents types de réflexivité, de différents intérêts de connaissance, devient un des enjeux centraux du terrain et de la production des connaissances. La « démystification» n'est plus le propre du sociologue.

\section{Généralisation de la double herméneutique comme compétence sociale et ses répercussions sur la reconnaissance de l'expertise sociologique}

Que ce soit par le travail de collecte des informations ou par l'organisation de synthèses susceptibles d'éclairer les différents protagonistes, le travail du chercheur peut se confondre avec celui des chargés de mission et des animateurs de territoire. Les occasions de méprises et de malentendus sont légion et les agacements fréquents. Un petit retour de terrain peut en témoigner. Lors de la phase d'entretiens qualitatifs dévolue à la collecte d'informations dans le cadre de Clim'Ability (cf. les articles dans 
ce même numéro), un des interlocuteurs expose son analyse de la situation afin d'illustrer les enjeux de sa mission. Cet exposé corrobore nos analyses. Il conforte le travail accompli les six derniers mois passés par l'équipe ${ }^{(23)}$. Quelques semaines après, nous déposons un projet en réponse à un appel d'offre auquel nous souhaitons l'associer en raison de son niveau d'expertise. À la lecture de notre proposition, ce dernier se sent trahi parce qu'il retrouve les informations qu'il nous a fournies. Selon son point de vue, nous nous sommes servis de son expertise que nous nous sommes appropriée; selon notre analyse, son expertise rejoint la nôtre établie par notre enquête et nous faisons preuve de correction en l'invitant à rejoindre notre projet.

\section{Le sociologue et la négociation de la commande institutionnelle}

Plutôt que de rester marginale cette situation est amenée à se généraliser en raison de ce que les pratiques de projet se rapprochent des pratiques de recherche par leur recours à la double herméneutique. La démocratisation de l'intervention sociale justifie le recours aux consultations multi-acteurs et à des processus par itération qui s'inspirent beaucoup de la production des connaissances socio-anthropologiques par immersion dans des terrains. En 2006, la démarche exploratoire du projet de thèse de Sophie Némoz portant sur les rapports sociaux à la qualité environnementale de l'habitat a retenu l'intérêt d'une institution: le Ministère français de l'écologie, de l'énergie, du développement durable et de l'aménagement du territoire. L'investigation a ainsi bénéficié du soutien du PUCA (Plan urbanisme construction et architecture) et de l'ADEME (Agence de l'environnement et de la maîtrise de l'énergie), deux organismes directement impliqués dans la promotion des normes afférentes à la durabilité des bâtiments. Le questionnement de cette catégorie spatiale a permis d'appréhender des expériences sociales variées et d'observer comment elle contribue en retour à structurer les groupes sociaux et les sociétés de manière différenciée ${ }^{(24)}$. Si la normalisation de l'habitat écologique a pu être déconstruite à travers l'enquête des coulisses de l'action publique en France, la comparaison menée dans plusieurs pays européens a aiguisé un regard distancié sur la dimension idéologique des dispositifs spatiaux $^{(25)}$. Non prévu dans le financement par projet, le décentrement ainsi opéré au cours du travail de thèse en sociologie a stimulé la recherche et élargi son horizon, avec près d'une centaine d'acteurs rencontrés à la fin de l'enquête. L'entretien biographique auprès de chacun d'eux s'est accompagné d'une visite commentée et photographiée de lieux, puis d'un dessin représentant leurs attributs et les pratiques sociales associées sur le plan esquissé. Les espaces intérieurs et extérieurs de l'habitat écologique ont ainsi été considérés à la fois comme un objet et un instrument d'investigation pour la

23 Julie Gobert, Alexandre Kudriavtsev, Paul Averbeck, Florence Rudolf, «Ladaptation des entreprises au changement climatique. Questionnements théoriques et opérationnels ", Revue d'Allemagne et des pays de langue allemande, 49/2 (2017): "L'année 1917, entre ancien et nouveau monde", p. 491-504.

24 Sophie Némoz, "L’habitat écologique: une construction sociale en voie de développement durable», Les annales de la recherche urbaine, $\mathrm{n}^{\circ} 106$ (2010), p. 16-25.

25 Sophie Némoz, "L'“éco-logis” politique: un dépaysement critique de l'habitat durable en Europe», Sciences de la société, nº 98 (2016): «Habitat durable: approches critiques», p. 31-43. 
sociologue ${ }^{(26)}$. Ils contribuent à éclairer la nature multidimensionnelle de cette innovation résidentielle et les décalages qu'elle instille entre les politiques, les professionnels et les habitants. La démarche "ancrée» dans le terrain a permis des rencontres avec des espaces, des personnes et des objets au-dedans et au-dehors des «Villas Urbaines Durables", ce programme national précurseur des constructions des éco-quartiers en France sur lesquelles de nombreux travaux se sont concentrés depuis lors. En ne limitant pas le champ de recherche à ce bornage, il s'est avéré que multiplier les points de vue, parfois antagonistes, permet de mieux comprendre la complexité des processus en action. L'enquête multi-située a ainsi déconstruit les dispositifs spatiaux dans lesquels les individus et les groupes bâtissent leur environnement et se différencient en regard des uns et des autres ${ }^{(27)}$.

\section{Face à la commande, faire le pique-bœuf}

La commande sociale peut être vécue comme un cadre particulièrement problématique, mais à l'inverse cette situation peut encore se retrouver instrumentée par le chercheur lui-même. Il s'ensuit que les chercheurs entrent de plus en plus en compétition avec les porteurs et animateurs de projet avec lesquels ils ne partagent pas toujours les mêmes obligations et exigences. Ainsi, Christophe Baticle profitera du dossier Natura 2000 pour mener deux lourdes enquêtes par questionnaires, entretiens non-directifs puis semi-directifs, observations et encore collecter des données dont seules les fédérations de chasseurs disposent. Celle de la Somme a alors, de 2003 à 2004 ${ }^{(28)}$, convenu de négocier la paix sociale sur le littoral picard en demandant un financement afin que soient évaluées les perceptions du dispositif européen de protection des habitats naturels chez les pratiquants cynégétiques, particulièrement nombreux dans le secteur et surtout suffisamment puissants pour constituer des acteurs incontournables. Le socio-anthropologue se fait alors pique-bœuf afin de pouvoir accéder à un public méfiant, sous couvert de ses institutions représentatives (fédérations et associations spécialisées de chasseurs de gibier d'eau). À la suite de cette expérience, on lui demandera de superviser les questionnaires destinés à trois autres sites de la moyenne vallée de la Somme, inspirés des enquêtes précédentes. Dès l'année suivante, en $2005^{(29)}$, c'est à nouveau une vaste enquête sociographique qui lui sera commandée afin de cadrer le

26 Sophie Némoz, L“éco-logis”, une innovation durable... Analyse sociologique de l'écologie résidentielle en France et au détour de la Finlande et de l'Espagne, thèse de doctorat en sociologie, Université Paris Descartes, 2009.

27 Sophie Némoz, «Le développement du logement durable: analyse d’une innovation en trois dimensions sociologiques ", Sciences de la société, $\mathrm{n}^{\circ} 81$ (2012) : "L'organisation entre normes et innovation", p. 137-157.

28 Christophe BATICLE, Chasse et environnement: implications réciproques? Étude cynégétique du site «Natura 2000: Estuaires et littoral picards» (PIC.01), rapport, Amiens, Université de Picardie Jules Verne (CEFRESS), avec la participation de la DIREN et du Conseil Régional de Picardie, 2003, 520 p.; Christophe BAtIClE, Chasse et environnement: implications réciproques? Étude cynégétique du site "Natura 2000: Marais Arrière littoraux picards»(PIC.02), rapport, Amiens, Université de Picardie Jules Verne (CEFRESS), avec la participation de la DIREN et du Conseil Régional de Picardie, 2004, $690 \mathrm{p}$.

29 Christophe Baticle, Les chasseurs de la Somme. Portrait social, rapport, Amiens, Université de Picardie Jules Verne (CEFRESS), 2005, 179 p. 
plan de gestion cynégétique départemental. Pas moins de 28000 adhérents à la fédération pourront être interrogés, avec un retour de 5420 questionnaires valides: une nouvelle moisson de données inenvisageable en dehors de ce contexte, dans lequel des questions éminemment politiques auront pu être intégrées à la dernière minute. Cette expérience motivera la fédération régionale du Nord-Pas-de-Calais à procéder de la même manière une dizaine d'années plus tard ${ }^{(30)}$. Par là, une masse d'informations a pu à nouveau être collectée (produite), mais l'image de chercheur affilié au monde cynégétique fut le coût à payer auprès de certains acteurs de la protection.

\section{Inductivité et déductivité: faire une place au terrain dans l'élaboration des hypothèses}

On se gardera de poursuivre une liste qui ne saurait se tarir sans faire référence à un dernier type de postures qui génère nécessairement des échanges avec le terrain, aboutissant à des formes d'hybridation des savoirs. Parmi l'ensemble des possibilités d'aborder une réalité sociale, un premier croisement se présente en effet quant à la relation aux théories. Pour simplifier à l'extrême, celles-ci peuvent être déterminantes, dès l'amont de la recherche, quant à la manière d'interroger la réalité, ce qui revient à assujettir, dans un premier temps au moins, la construction de «l'objet » à une grille de lecture théorique retenue au préalable. On a regroupé ces approches dans l'appellation "hypothético-déductivité», les déductions résultant des hypothèses théoriques. Ici, on peut penser que le chercheur détient la maîtrise de son "cadre théorique», lequel va l'orienter dans sa «collecte des données». À l'inverse, si c'est le terrain qui guide une quête sans objet prédéfini par avance, sur un mode inductif, les questions auront tendance à émerger progressivement des investigations entreprises in situ, reportant le travail de théorisation à une étape ultérieure, à partir des informations issues des interactions entre le chercheur et ses rencontres. Ainsi, dans l'hypothético-inductivité ce sont les hypothèses qui sont censées succéder à l'observation du terrain, bien que dans la réalité de la recherche la césure entre les deux approches soit moins étanche qu'il n'y paraît, un cadre théorique pouvant par exemple être présent, de façon plus ou moins consciente, au cours d'une investigation inductive. Ceci étant, la démarche implique, de la part du chercheur, une attention soutenue aux «théories» produites par les acteurs du terrain qui, «prises au sérieux» en quelque sorte, viennent alimenter les outils de la socio-anthropologie. Les données elles-mêmes sont ainsi produites plus que collectées. Tel le Fabrice de Stendhal à la bataille de Waterloo ${ }^{(31)}$, le chercheur est un naï, ou tout au moins joue les naïs par ses questions absurdes, prenant ses distances avec ses propres constats de visu: il voit en doutant de ses interprétations. Même en étant intégré au sein des hussards de Napoléon I ${ }^{\text {er }}$, il n’est pas certain de sa défaite face à la coalition anglo-prussienne.

30 Christophe Baticle, Sociographie des chasseurs du Nord-Pas-de-Calais, rapport, Amiens, Habiter le Monde, 2015, 145 p.

31 Stendhal, La chartreuse de Parme, 1839. 


\section{Le récit, un vecteur puissant de transformation sociale}

La production de sens et par un pas de plus la mise en récit demeure in fine un des enjeux essentiels de la transformation sociale, entre reproduction matérielle et reproduction symbolique de la société. À l'instar des travaux de Lepenies ${ }^{(32)}$ sur la raison sociologique, cette dernière a été tout autant stimulée par la raison littéraire que par la raison scientifique. La raison littéraire n'a pas attendu les avancées de la recherche en sciences sociales pour reconnaître la puissance du récit comme force sociale. Dans le roman Neige d'Orhan Pamuk ${ }^{(33)}$, l'imbroglio susceptible de résulter de la généralisation de la double herméneutique (action par la production de récit, le récit comme levier du projet, etc.) conduit l'intrigue qui se déroule aux confins orientaux de la Turquie où l'arrivée d'un journaliste enquêteur des suicides de jeunes filles voilées précipite le cours des événements.

À l'inverse, sur les terrains de l'enquête sociologique, de multiples situations peuvent être mises en exergue pour illustrer ces phénomènes d'émergence d'une réalité «révélée» par la recherche. Les mouvements régionalistes en témoignent. On sait par exemple que le massif du Vercors, bien connu pour son Parc naturel régional, l'un des tout premiers créés en 1970, et surtout haut-lieu de la Résistance française pendant la Seconde Guerre mondiale, émergea notamment des travaux de collègues géographes de l'École de Grenoble, qui en firent une de leurs terres de prédilection ${ }^{(34)}$. Par ailleurs, cette stimulation entre la recherche et la littérature peut concerner les concepts. Pour illustration, celui de chronotope, qui visait initialement à repérer des espaces-temps caractéristiques du roman, se retrouve aujourd'hui appliqué à des terrains de recherche. Philippe Hanus et Christophe Baticle ${ }^{(35)}$ voient ainsi dans la meule de charbon de bois un chronotope propice à la réinvention du monde chez les néocharbonniers qui ont patrimonialisé cette pratique.

Le travail sociologique n'échappe pas à l'immersion sociale, par l'entremise des négociations qui permettent au sociologue d'être apprivoisé par le terrain qu'il vise. Au même titre que l'ethnologue mis en scène par Georges Perec dans La vie mode d'emploi ${ }^{(36)}$, où il est question d'un ethnologue qui cherche des terrains bienveillants et durables ou des carrières décrites par l’École de Chicago, les sociologues négocient leur place dans des situations qu'ils tentent de définir à l'instar des acteurs qui en sont les "propriétaires». Si on ne peut faire abstraction des compétences

32 Wolf Lepenies, Les trois cultures. Entre science et littérature, l'avènement de la sociologie (1985), traduction de H. Plard, Paris, Éditions de la Maison des sciences de l'Homme, 1990.

Orhan Pamuk, Neige (2002), trad. de Jean-François Perouse, Paris, Gallimard, 2005.

Anne SGARD, «L'invention d'un territoire», L'Alpe, hors série: "Vercors en questions », pour les 30 ans du PN-Alpes, avec le soutien du Parc naturel régional du Vercors, le Ministère de l'aménagement du territoire et de l'environnement, le Conseil régional Rhône, le Conseil général de la Drôme, le Conseil général de l'Isère, 2001, p. 42-53.

Christophe BAticle, Philippe Hanus, «Les nuits contestataires des néo-charbonniers du Vercors: un chronotope forestier au service d'une hétérotopie», Journal of Alpine Research/Revue de géographie alpine (en ligne), 106/1 (2018), mis en ligne le 18 avril 2018, http://journals.openedition.org/rga/3958; DOI: $10.4000 /$ rga.3958. 
du sociologue dans ces épreuves, il n'en demeure pas moins qu'elles témoignent de l'ouverture sociale d'un terrain, de son hospitalité à l'égard de cet acteur d'un type un peu particulier qu'est le sociologue. Le récit de ces apprivoisements réciproques participe de la connaissance d'une cause et des collectifs qui la cultivent. Il nous informe du rapport que ces derniers entretiennent à la réflexivité. Ce constat apporte un nouvel éclairage à la réception et participe à l'acceptation sociale des analyses sociologiques.

\section{Conclusion : pour une sociologie de l'ouverture à l'altérité}

Le recensement des figures susceptibles d'inspirer une grammaire de la double herméneutique demeure à établir. Nous espérons avoir rendu compte de l'intérêt et de la richesse de ce programme de recherche: les retours de terrains relayés par des œuvres littéraires semblent une piste intéressante à explorer! Quid cependant du traitement des non-humains par rapport aux terrains dont les sociologues sont familiers et que l'essor des humanités environnementales rend d'autant plus actuel?

À l'aune de cette discussion, on mesure que l'irruption au creux des recherches sociologiques de nouvelles entités, relevant du vivant, correspond à un redoublement des enjeux à soulever. Comment intégrer dans les recherches des entités relevant de la technique ou du vivant? Si l'activité scientifique rechigne à composer avec des humains et des publics non initiés, qui le lui rendent bien ainsi que cet article s'est employé à le montrer, qu'en est-il des non-humains? Les travaux regroupés sous l'expression d'acteur-réseau ont tenté de relever ce défi ${ }^{(37)}$. Ils y sont parvenus en s'intéressant aux relations plutôt qu'aux entités, renouant de la sorte avec un des fondamentaux de la sociologie. Ce qui fait l'acteur, ce n'est pas son appartenance à l'humanité ou uniquement cette dernière, mais les équipements dont il est paré. En raisonnant de la sorte, l'intelligibilité d'une situation procède des entités en présence et de leur agencement. C'est l'acteur-réseau qui est déterminant et qui confère à une situation son agentivité et par conséquent son aptitude à s'organiser selon une trajectoire plutôt qu'une autre. Ce paradigme permet d'élargir la société à de nombreux êtres qui n'y occupaient qu'une place marginale à titre d'objets par définition inertes. Ces derniers, qualifiés de non-humains, vivants ou non, entrent en relation avec d'autres entités, dans des associations qui activent respectivement les êtres en présence. C'est à cet égard qu'on peut qualifier cette sociologie de constructiviste. Ce qualificatif englobe cependant des approches diverses allant jusqu'à s'exclure. Il ne s'agit pas d'un plaidoyer en faveur d'un relativisme radical, mais en faveur d'une recherche qui compose à partir de la situation. Ainsi lorsqu'une chercheuse comme Vinciane Despret invite à "penser comme un rat», c'est en vue de résister à une science qui produit des artefacts qui nient la personnalité des entités qui l'intéressent.

37 Bruno Latour, Petites leçons de sociologie des sciences, Paris, Seuil, 1996; Corinne BeCk, Élisabeth RÉMY, «La Loutre d'Europe (Lutra lutra). Observations profanes et données normalisées de l'inventaire naturaliste», Études Rurales, janvier-juin 2005, n 195, p. 43-58; Christelle Gramaglia, «Des poissons aux masses d'eau: des usages militants du droit pour faire entendre la parole d'êtres qui ne parlent pas", Politix, 83 (2008), p. 133-153; Élisabeth RÉмy, "Méthode indiciaire et prises photographiques. Le cas du suivi de la loutre», Natures Sciences Sociétés, 14 (2006), p. 144-153. 
Cette approche n'étant qu'une des étapes et non des moindres de la recherche qui vise in fine à penser avec eux.

À propos de Barbara Smuts qui a travaillé avec les babouins, elle précise:

"Il ne s'agit pas de savoir, de l'intérieur, ce que pense un babouin, ce que le "penser comme" pourrait laisser entendre. [...] Devrais-je modifier mon titre? Mais il s'agit d'un trajet et de ce que j'y apprends, et ce trajet a importé; il ne faut pas l'effacer. Juste se rappeler sur quel chemin on marche. Car c'est bien cela qui est au cœur de la réussite de Smuts, et de son propre trajet: arriver à reconnaître que c'est "penser avec" qui est à la fois l'enjeu et la condition de la recherche» ${ }^{(38)}$.

Sans plonger davantage dans ce magnifique petit ouvrage, témoin des efforts entrepris par l'INRA pour ouvrir ses chercheurs à des recherches inédites pour ne pas dire insolites, cette invitation à composer avec l'altérité se pose de manière finalement pas si différente qu'avec des êtres techniques. La sociologie des sciences s'est employée, par le détour de la théorie de l'acteur-réseau, à considérer les situations de recherche comme des situations d'entre-capture. Cette définition extrait/extirpe la méthodologie du rôle ingrat qui lui était dévolu en raison de l'absence de créativité propre aux protocoles. Or, à partir du moment où la méthodologie est associée à la genèse d'un intéressement réciproque, soit à l'art d'interpeller un autre, elle gagne des lettres de noblesse. Les réflexions à propos des méthodes s'apparentent alors à un exercice de diplomatie. Elles précisent le sens du propos de Bruno Latour lorsqu'il rapproche la science de la politique ${ }^{(39)}$. Faire entrer la nature en politique, pour reprendre un titre célèbre de son œuvre, s'entend également comme l'art d'intéresser des entités hors champ afin de les constituer en partenaires d'un monde commun. Un tel art prémunit non seulement les chercheurs de se transformer en tortionnaires des animaux de laboratoires pour les faire entrer dans des artefacts qui n'apprennent rien sur ces derniers $^{(40)}$, mais il sert le développement d'une science responsable. S'exercer à «penser avec eux » c'est s'interdire de trahir, de délirer, de détruire au nom d'une science arrogante et malhonnête.

Cette précision sur de nouvelles manières de faire science en s'ouvrant à son objetsujet d'étude de manière à générer de la communication, peut-être davantage au sens luhmannien d'une connexion structurelle qu'au sens habermassien d'une intersubjectivité, apporte une précision quant au caractère politique de l'activité scientifique. Faire preuve de qualité de terrain, c'est faire preuve de diplomatie, laquelle peut, par un pas de plus, s'ouvrir aux arts plastiques et communicationnels. Ce sont

38 Vinciane Despret, Penser comme un rat, Nancy, Éditions QUAE (coll. Sciences en questions), 2009, p. 7-48.

39 Bruno Latour, Politiques de la nature. Comment faire entrer les sciences en démocratie, Paris, La Découverte, 1999.

40 «Les behavioristes, John Watson en particulier, ont en fait bel et bien envisagé l'influence de ces caractères perceptifs optiques, tactiles et olfactifs. Je n'irais toutefois pas jusqu'à affirmer qu'ils les ont examinés. À moins de confondre le terme “examiner” avec celui de "neutraliser". Car c'est bien ce qu'a fait Watson, dans une procédure qui, si l'on pense qu'elle s'apparente à l'examen, devrait conduire les malades chez un tortionnaire plutôt que chez leur médecin: il a retiré au rat ses yeux, son bulbe olfactif et ses vibrisses, essentiels au sens du toucher chez le rat, avant de le lancer à la découverte du labyrinthe» (V. Despret, note 36, p. 31-32). 
exactement ces glissements qu’a opérés Bruno Latour au cours des dernières années ainsi qu'en témoignent son dernier spectacle Inside et ses expositions au Zentrum für Kunst und Medien (ZKM) ${ }^{(41)}$.

\section{Résumé}

À partir de leurs expériences de terrain, principalement centrées sur la socio-anthropologie de l'environnement, les quatre co-auteurs de cet article se proposent de revenir sur les modalités qui ont déterminé leurs conditions de recherche, en particulier les modes opératoires par lesquels se sont négociées, y compris implicitement, leurs entrées sur des terrains variés. De ce point de départ est envisagée une "grammaire» relationnelle entre les milieux étudiés et la recherche scientifique. La réflexivité des acteurs rencontrés in situ les conduit à se rapprocher de la double herméneutique d'Anthony Giddens, pour lequel le terrain agit en retour sur les productions sociologiques, dans un mouvement d'allers et de retours continu. Les figures de l'étranger (Simmel) ou du chasseur de mythes (Élias) ne sont ici que des étapes pour progresser vers des configurations, entre hypothético-déductivité et hypothético-inductivité, dont la littérature ou la nature fournissent les métaphores. De ces esquisses grammaticales se tissent quelques manières de conjuguer le terrain, dont on peut espérer de nouveaux développements issus d'autres expériences.

41 Centre d'art et de technologie des médias de Karlsruhe. 